\title{
Angular dependent NEXAFS study of the molecular orientation of PTCDA multilayers on Au (111) surface
}

\author{
CAO Liang ${ }^{1}$, ZHANG WenHua ${ }^{1}$, HAN YuYan ${ }^{1}$, CHEN TieXin ${ }^{1}$, ZHENG ZhiYuan $^{1}$, WAN Li $^{1}$, \\ XU FaQiang ${ }^{*}$, IBRAHIM Kurash ${ }^{2}$, QIAN HaiJie ${ }^{2} \&$ WANG JiaOu ${ }^{2}$ \\ ${ }^{1}$ National Synchrotron Radiation Laboratory, School of Nuclear Science and Technology, University of Science and Technology of China, Hefei \\ 230029, China: \\ ${ }^{2}$ Beijing Synchrotron Radiation Laboratory, Institute of High Energy Physics, Chinese Academy of Sciences, Beijing 100039, China
}

Received April 22, 2011; accepted June 6, 2011

The molecular orientation of perylene-3,4,9,10-tetracarboxylic acid dianhydride (PTCDA) multilayers adsorbed on Au (111) surface has been investigated using angular dependent $\mathrm{O} K$-edge near edge X-ray absorption fine structure (NEXAFS) spectroscopy. The significant angular dependence of important resonant structures $\left(\pi^{*}\right.$ and $\left.\sigma^{*}\right)$ reveals that PTCDA molecules adopt an ordered geometry on the substrate surface. The average tilt angle of the PTCDA molecular planes is $27^{\circ} \pm 10^{\circ}$ from the $\mathrm{Au}(111)$ surface.

NEXAFS, perylene-3,4,9,10-tetracarboxylic acid dianhydride, organic electronics, molecular orientation

Citation: Cao L, Zhang W H, Han Y Y, et al. Angular dependent NEXAFS study of the molecular orientation of PTCDA multilayers on Au (111) surface. Chinese Sci Bull, 2011, 56: 3575-3577, doi: 10.1007/s11434-011-4792-5

In recent years, organic semiconductors have been widely used as active components in many electronic and optoelectronic devices [1-3]. In most of these devices, molecule/ metal interfaces are ubiquitous. It is well known that molecular morphology, organization and electronic structure at interfaces play important roles in determining device performance [4]. In particular, molecular orientation is likely to influence interfacial electronic properties [5,6]. Therefore, it is of importance to prepare organic thin films containing ordered molecules to better understand and enhance device performance.

PTCDA $\left(\mathrm{C}_{24} \mathrm{O}_{6} \mathrm{H}_{8}\right.$, chemical structure shown in Figure 1) is an n-type organic semiconductor with an optical transport band gap of $2.2 \mathrm{eV}$. This gap can be modified by substituting hydrogen or oxygen atoms for various functional groups. PTCDA and its derivatives [7] are widely applied in the fields of organic photovoltaic cells (OPVCs) and organic light emitting diodes (OLEDs) [8].

\footnotetext{
*Corresponding author (email: fqxu@ ustc.edu.cn)
}

PTCDA molecules can grow in an ordered fashion on various substrates by organic molecular beam deposition (OMBD) [1,9]. The adsorption behavior of ultrathin layers of PTCDA (e.g. sub-monolayer, monolayer and bilayers) on $\mathrm{Au}$ (111) has been investigated by scanning tunneling microscopy (STM) [10-12]. However, less effort has been devoted to multilayers because of the limitations of STM. Previously, we reported a qualitative analysis of the angular dependent NEXAFS spectra at the C $K$-edge obtained for PTCDA molecules adsorbed on Au (111)/mica surface [13]. Angular dependent NEXAFS has been found to be a powerful tool for characterizing the adsorption geometry of planar aromatic molecules on a solid substrate [14]. In addition, NEXAFS is also useful for studying absorption sites $[15,16]$ and bond lengths $[12,17]$ between adsorbates and substrate atoms, although these were not investigated in this work. In this paper, quantitative analysis of the average molecular tilt angle for PTCDA multilayers adsorbed on $\mathrm{Au}$ (111) single crystal surface was performed by angular dependent NEXAFS at the $\mathrm{O} K$-edge. 


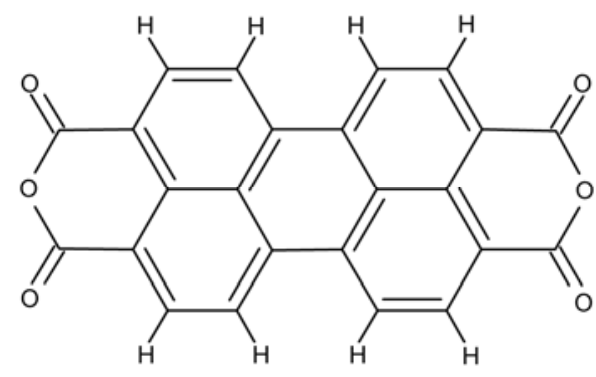

Figure 1 The molecular structure of PTCDA.

\section{Experimental}

NEXAFS measurements were carried out at the Photoemission Spectroscopy Endstation at the 4B9B beamline of the Beijing Synchrotron Radiation Facility of the Institute of High Energy Physics, Chinese Academy of Sciences. The O $K$-edge NEXAFS spectra were performed in total electron yield (TEY) mode with a photon energy resolution of 500 meV. To eliminate the effect of fluctuation of the incident beam intensity, all spectra were first normalized to the incident beam flux (monitored by refocusing mirror $I_{0}$ ) and later normalized to the same edge jump [18].

$\mathrm{Au}$ (111) single crystal was cleaned in situ by repeated cycles of sputtering and annealing. The cleanliness of the crystal surface was checked by Auger photoemission spectroscopy (AES) and photoemission spectroscopy (PES). PTCDA molecules, which were thoroughly degassed for several hours, were deposited onto the clean Au (111) substrate at room temperature in an ultrahigh vacuum (UHV) chamber with a base pressure better than $1 \times 10^{-8}$ torr. A deposition rate of $\sim 2 \mathrm{ML} / \mathrm{min}$ was precalibrated using a quartz crystal microbalance (QCM) at the Surface Physics Endstation of the National Synchrotron Radiation Laboratory.

\section{Results and discussion}

In molecular systems, NEXAFS monitors resonant electronic transitions from the inner shell of specific atomic species (C $1 s, \mathrm{O} 1 s$ or $\mathrm{N} 1 s)$ to unoccupied molecular orbitals or states ( $\pi^{*}$ and $\sigma^{*}$ orbitals). It has been well established that the resonant intensity is enhanced if the electric field vector $\boldsymbol{E}$ of synchrotron light polarization is parallel to the direction of the molecular orbital, and the intensity of the resonance is suppressed if $\boldsymbol{E}$ is perpendicular to the orbital direction [14]. For planar $\pi$-conjugated molecules like PTCDA, $\sigma^{*}$ and $\pi^{*}$ orbitals are essentially oriented in-plane and out-of-plane, respectively. Therefore, the molecular orientation within a layer can easily be derived from the relationship between the resonant intensities and incident angle of light.

Angular dependent $\mathrm{O} K$-edge NEXAFS spectra of PTCDA multilayers (nominal coverage of $10 \mathrm{ML}$ ) adsorbed on $\mathrm{Au}$ (111) surface are shown in Figure 2. Sharp resonant peaks below $538 \mathrm{eV}$ arise from $\mathrm{O} 1 \mathrm{~s} \rightarrow \pi^{*}$ transitions, whereas resonances located at higher energy originate from O $1 \mathrm{~s} \rightarrow \sigma^{*}$ transitions. The strongest $\pi^{*}$ resonant peak is asymmetric with a dominant component (labeled 1) and a subtle shoulder (labeled 2) at slightly higher photon energy. Peaks 1 and 2 are assigned to the transitions of carbonyl oxygen atoms $(\mathrm{C}=\mathrm{O})$ to the lowest unoccupied molecular orbital (LUMO) and higher orbitals (LUMO+1 and LUMO+2), respectively [19]. Resonant peak 3 is attributed to the transition from the bridging $\mathrm{O}$ atoms $(\mathrm{C}-\mathrm{O}-\mathrm{C})$ to the LUMO+1 (and perhaps LUMO+2 and LUMO+3) [19].

The spectra show that the intensity of the $\pi^{*}$ resonances vary significantly with incident angle, and reach maximum and minimum values at $\theta=10^{\circ}$ and $\theta=90^{\circ}$, respectively. The $\sigma^{*}$ resonances show the opposite behavior. This polarization dependence directly suggests the formation of ordered layers of PTCDA molecules on the Au (111) surface.

In terms of quantitative analysis, the intensity of $\pi^{*}$ resonances $\left(I_{\pi^{*}}\right)$ at various incident angles $(\theta)$ can be expressed as follows [14]:

$I_{\pi^{*}}=C P\left(\sin ^{2} \alpha \sin ^{2} \theta+2 \cos ^{2} \alpha \cos ^{2} \theta\right)+C(1-P) \sin ^{2} \alpha$,

where $C$ is a normalization constant, $P$ is the linear polarization factor $(0.75)$, and $\alpha$ is average tilt angle of $\pi^{*}$ vector orbital versus the surface normal. In multilayers, the interaction between the molecules and substrate is too weak to affect the direction of $\pi^{*}$ orbitals. Therefore, $\alpha$ is equal to the average tilt angle of the molecular planes of PTCDA with respect to the surface plane (see Figure 2 inset).

The integrated intensities of the first two and first three $\pi^{*}$ resonances as a function of $\theta$ together with theoretical

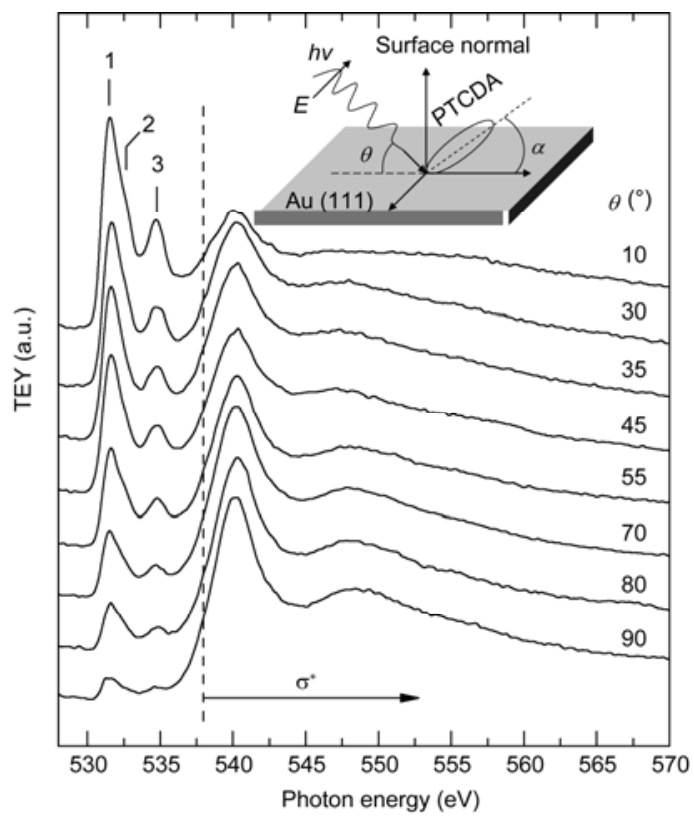

Figure 2 Angular dependent $\mathrm{O} K$-edge NEXAFS spectra of PTCDA multilayers on $\mathrm{Au}$ (111) surface. Incident angles are designated with respect to the surface plane. The inset shows the geometry of the experiment. 


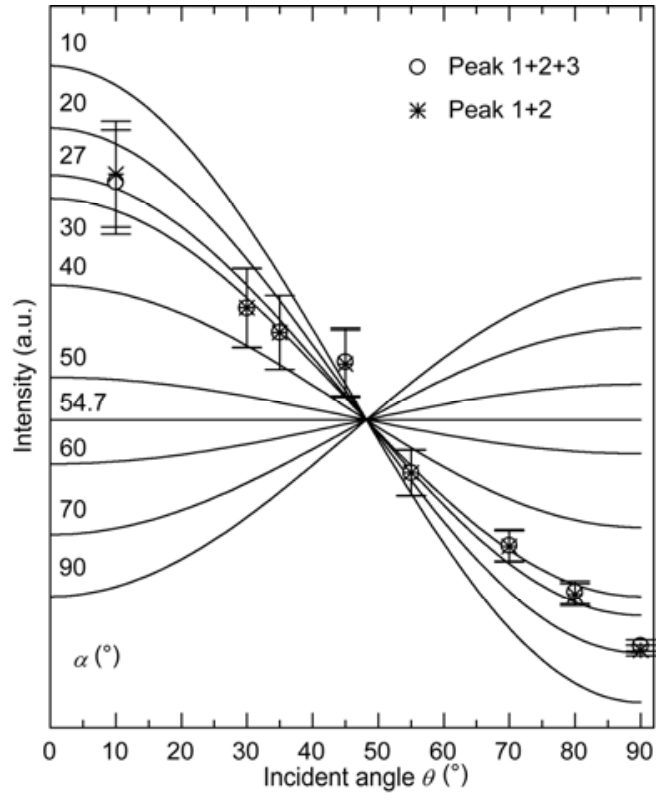

Figure 3 The integrated intensities of the first two (stars) and first three (open circles) resonances against $\theta$ for multilayer thin films of PTCDA. The solid lines represent the theoretical angular dependences for various tilt angles. Normalization constants are $C_{1+2}=16.07$ and $C_{1+2+3}=24.13$.

curves for different tilt angles (solid lines) obtained using eq. (1) are presented in Figure 3. The error bars correspond to uncertainties introduced by the normalization and integral, and are estimated to be $10 \%$ of the intensity [20]. The average tilt angle $\alpha$ for molecular planes of PTCDA on Au (111) surface was determined to be $27^{\circ} \pm 10^{\circ}$. The overall uncertainty of $10^{\circ}$ takes into account fitting assumptions, fitting error, the degree of polarization of the X-ray beam, and the misalignment between the surface and reference manipulator orientation [21].

\section{Conclusions}

In conclusion, PTCDA multilayers were deposited on $\mathrm{Au}$ (111) surface by OMBD. Angular dependent O K-edge NEXAFS spectroscopy was applied in situ to investigate the molecular orientation at the PTCDA/Au (111) interface. It is concluded from quantitative analysis of the angular dependence that PTCDA molecules are ordered within the film with an average tilt angle of $27^{\circ} \pm 10^{\circ}$ from the substrate surface.

The authors thank Dr. QI Dongchen from the National University of Singapore for helpful discussion. This work was supported by the National Natural Science Foundation of China (10975138, 10505019 and 10775126) and the Scientific Research Foundation of the Graduate School of the University of Science and Technology of China.

1 Forrest S R. Ultrathin organic films grown by organic molecular beam deposition and related techniques. Chem Rev, 1997, 97: 1793-1896

2 Mathine D L, Woo H S, He W, et al. Heterogeneously integrated organic light-emitting diodes with complementary metal-oxide-silicon circuitry. Appl Phys Lett, 2000, 76: 3849-3851

3 Liu J Q, Chen S F, Chen L, et al. Organic/polymeric memory and their switching mechanisms (in Chinese). Chinese Sci Bull (Chinese Ver), 2009, 54: 3420-3432

4 Barth J V, Costantini G, Kern K. Engineering atomic and molecular nanostructures at surfaces. Nature, 2005, 437: 671-679

5 Xu Z X, Roy V A L, Liu Z T, et al. Imporatance of molecular alignment for organic photovoltaic devices. Appl Phys Lett, 2010, 97 : 163301

6 Chen W, Qi D C, Huang Y L, et al. Molecular orientation dependent energy level alignment at organic-organic heterojunction interfaces. J Phys Chem C, 2009, 113: 12832-12839

7 Sun J Z, Yang X G, Wang M. Thin films of porphyrin-perylene molecular array fabricated by electrophoresis methodology. Chinese Sci Bull, 2005, 50: 2157-2160

8 Guo Y L, Yu G, Liu Y Q. Functional organic field-effect transistors. Adv Mater, 2010, 22: 4427-4447

9 Eremtchenko M, Schaefer J A, Tautz F S. Understanding and tuning the epitaxy of large aromatic adsorbates by molecular design. Nature, 2003, 425: 602-605

10 Chizhov I, Kahn A, Scoles G. Initial growth of PTCDA on Au (111): A scanning tunneling microscopy study. J Cryst Growth, 2000, 208: 449-458

11 Mannsfeld S, Toerker M, Schmitz-Hübsch T, et al. Combined LEED and STM study of PTCDA growth on reconstructed Au (111) and Au (100) single crystals. Org Electron, 2001, 2: 121-134

12 Schmitz-Hübsch T, Fritz T, Sellam F, et al. Epitaxial growth of PTCDA on Au (111): A STM and RHEED study. Phys Rev B, 1997, 55: 7972-7976

13 Cao L, Zhang W H, Chen T X, et al. The molecular orientation and electronic structure of 3,4,9,10-perylene tetracarboxylic dianhudride grown on Au (111). Acta Phys Sin, 2010, 59: 1681-1688

14 Stöhr J. NEXAFS Spectroscopy. Berlin: Springer, 1996

15 Tang J C, Shen J F, Feng X S, et al. Determination of HCOO-Cu (100) structure by MSC method of NEXAFS. Chinese Sci Bull, 1990, 35: 806

16 Zhang W T, Li H B, Wang Y M, et al. Correlation between structure and hardness of magnetron sputtering deposited $\mathrm{CN}_{x}$ film. Chinese Sci Bull, 1999, 44: 1149-1152

17 Tang J C, Fu S B, Ji H, et al. Multiple scattering cluster theory for X-ray absorption fine structure. Sci China Ser A, 1992, 35: 965-976

18 Outka D A, Stöhr J. Curve fitting analysis of near-edge core excitation spectra of free, adsorbed, and polymeric molecules. J Chem Phys, 1988, 88: 3539-3554

19 Taborski J, Väterlein P, Dietz H, et al. NEXAFS investigations on ordered adsorbate layers of large aromatic molecules. J Electron Spectrosc Relat Phenom, 1995, 75: 129-147

20 de Jong M P, Friedlein R, Sorensen S L, et al. Orbital-specific dynamic charge transfer from $\mathrm{Fe}(\mathrm{II})$-tetraphenylporphyrin molecules to molybdenum disulfide substrates. Phys Rev B, 2005, 72: 035448

21 Sing M, Meyer J, Hoinkis M, et al. Struncural vs electronic origin of renormalized band widths in TTF-TCNQ: An angular dependent NEXAFS study. Phys Rev B, 2007, 76: 245119

Open Access This article is distributed under the terms of the Creative Commons Attribution License which permits any use, distribution, and reproduction in any medium, provided the original author(s) and source are credited. 